NOTICE WARNING CONCERNING COPYRIGHT RESTRICTIONS:

The copyright law of the United States (title 17, U.S. Code) governs the making of photocopies or other reproductions of copyrighted material. Any copying of this document without permission of its author may be prohibited by law. 


\title{
Higher-Dimensional Voronoi Diagrams in Linear Expected Time
}

\author{
Rex A. Dwyer * \\ 11 February 1988 \\ CMU-CS-88-100
}

Keywords. Voronoi diagrams, computational geometry, average-case analysis of algorithms, polytopes, geometric probability.

\begin{abstract}
A general method is presented for determining the mathematical expectation of the combinatorial complexity and other properties of the Voronoi diagram of $n$ independent and identically distributed points. The method is applied to derive exact asymptotic bounds on the expected number of simplices of the Voronoi diagram of points chosen from the uniform distribution on the interior of a $d$-dimensional ball; it is shown that in this case, the complexity of the diagram is $\Theta(n)$ for fixed $d$. An algorithm for constructing the Voronoi diagram is presented and analyzed using the new method. The algorithm is shown to require only $\Theta(n)$ time on average for random points from a $d$-ball assuming a real-RAM model of computation with a constant-time floor function. This algorithm is asymptotically faster than any previously known and optimal in the average-case sense.
\end{abstract}

This research was sponsored by the National Science Foundation under contract number CCR-8658139. The views and conclusions contained in this document are those of the authors and should not be interpreted as representing the official policies, either expressed or implied, of the National Science Foundation or the US Government. 


\title{
Higher-Dimensional Voronoi Diagrams in Linear Expected Time
}

\author{
Rex A. Dwyer * \\ 11 February 1988
}

\section{Introduction}

The Voronoi diagram is a natural and intuitively appealing structure. First conceived by the mathematician Voronoi [28], it has been reinvented by researchers in several fields; in particular, meteorologists associate the two-dimensional version with the name Thiessen [26], and physicists honor Wigner and Seitz [30] for the three-dimensional version. It has been used by geologists, foresters, agriculturalists, medical researchers, geographers, crystallographers, and astronomers. Within the domain of the mathematical sciences, it is applied to simulate differential equations by finite element methods, to interpolate surfaces in geometric modeling systems, and to solve geometric problems such as finding Euclidean minimum spanning trees and largest empty circles. (Avis \& Bhattacharya [1] present an extensive list of references for applications.)

The Voronoi diagram of a set of points - called sites - is a partition of $\mathbf{R}^{d}$ that assigns a surrounding polytope of "nearby" points to each of the sites. More rigor is supplied by the following definition.

Definition 1 The (nearest-site) Voronoi diagram of the set $X_{n}=\left\{x_{1}, x_{2}, \ldots, x_{n}\right\}$ of $n$ sites in $\mathbf{R}^{d}$ is the set of $n$ convex regions $V_{i}=\left\{x \mid \forall j: \operatorname{dist}\left(x, x_{i}\right) \leq \operatorname{dist}\left(x, x_{j}\right)\right\}$ for $1 \leq i \leq n$.

Each region is a $d$-polytope containing the points lying nearer to the site in its interior than to any other site. The straight-line dual of the Voronoi diagram in the plane is called the Delaunay triangulation. In the planar case, sites $x_{i}$ and $x_{j}$ are joined by an edge in Delaunay triangulation if and only if $\nu_{i}$ and $\nu_{j}$ share an edge. In $d$ dimensions, sites $x_{i_{0}}, x_{i_{1}}, \ldots, x_{i_{k}}$ define a $k$-face of the Voronoi dual if and only if $V_{i_{0}}, V_{i_{1}}, \ldots, V_{i_{k}}$ share a $(d-k)$-face in the Voronoi diagram. If, as is assumed in the sequel, no $d+2$ sites fall on the same hypersphere, the dual partitions the convex hull of $X_{n}$ into $d$-simplices. Each of these simplices has the property that no points of $X_{n}$ lie in the interior of the hypersphere defined by its $d+1$ vertices, for the center of the hypersphere must be shared by the $d+1$ corresponding Voronoi regions; we say that the hypersphere is empty or site-free. The Voronoi diagram can be constructed easily from its dual and vice versa.

One may also define a furthest-site Voronoi diagram.

\footnotetext{
"This research was sponsored by the National Science Foundation under contract number CCR-8658139. Author's address: Computer Science Dept., Carnegie-Mellon University, Pitteburgh, Pennsylvania 15213 
Definition 2 The furthest-site Voronoi diagram of the set $X_{n}=\left\{x_{1}, x_{2}, \ldots, x_{n}\right\}$ of $n$ sites in $\mathbf{R}^{d}$ is the set of $n$ convex regions $W_{i}=\left\{x \mid \forall j: \operatorname{dist}\left(x, x_{i}\right) \geq \operatorname{dist}\left(x, x_{j}\right)\right\}$ for $1 \leq i \leq n$.

Region $W_{i}$ contains the points lying further from site $x_{i}$ than from any other site. Only sites that are vertices of the convex hull of $X_{n}$ have non-empty furthest-site Voronoi regions. The dual of the furthest-site Voronoi diagram also partitions the convex hull of $X_{n}$ into simplices; the vertices of these simplices determine hyperspheres that each contain all the sites of $x_{n}$.

Many researchers have considered Voronoi-diagram construction. The author has surveyed previous work on the two-dimensional case elsewhere [9]. The construction of three-dimensional diagrams has been addressed by Browstow et al. [6], Finney [12], and Tanemura et al. [24]

Bowyer [4] and Watson [29] describe algorithms for higher dimensions, but neither analyzes his algorithm rigorously. Bowyer argues heuristically that his algorithm requires $O\left(n^{1+1 / d}\right)$ time on average for points uniform in a $d$-dimensional hypercube, and cites some empirical evidence to support the claim. Avis and Bhattacharya's algorithms for the Voronoi diagram and its dual [1] rely heavily on the simplex method for linear programming; since this method has exponential worstcase running time, they focus mainly on experimental studies of their algorithms' performance and of the expected complexity of the diagrams for points distributed uniformly in the unit hypercube.

A pleasing connection between nearest- and furthest-site Voronoi diagrams in $d$ dimensions and convex hulls in $d+1$ dimensions allows any convex-hull algorithm to be used to construct Voronoi diagrams. This mapping, first observed by Brown [5], is restated here in a form due to Guibas \& Stolf [14]. If $a$ is a $d$-vector and $b$ is scalar, let $a \star b$ be the $(d+1)$-vector $\left(a^{(1)} a^{(2)}, \ldots, a^{(d)}, b\right)$, and let $\lambda: \mathbf{R}^{d} \rightarrow \mathbf{R}^{d+1}$ be the "lifting function" defined by

$$
\lambda(x)=x \star\langle x, x\rangle
$$

where $\langle x, y\rangle$ denotes the inner product. The range of $\lambda$ is the surface of a $(d+1)$-dimensional paraboloid of revolution. The image of a $d$-sphere is the intersection of a hyperplane with the paraboloid and vice versa. To verify this, we note that $x$ lies within the $d$-sphere centered at $p$ with radius $r$ if and only if $\langle x-p, x-p\rangle \leq r^{2}$ and apply the bilinearity of the inner product:

$$
\begin{aligned}
& \langle x-p, x-p\rangle \leq r^{2} \\
\Leftrightarrow & \langle x, x\rangle-2\langle p, x\rangle+\langle p, p\rangle \leq r^{2} \\
\Leftrightarrow & \langle((-2 p) \star 1),(x \star\langle x, x\rangle)\rangle \leq r^{2}-\langle p, p\rangle \\
\Leftrightarrow & \langle((-2 p) \star 1), \lambda(x)\rangle \leq r^{2}-\langle p, p\rangle .
\end{aligned}
$$

It is clear from the last line that $\lambda(x)$ lies both on the paraboloid and in some halfspace $\langle a, x\rangle \leq b$ in $\mathbf{R}^{d+1}$. Now suppose that the function $\lambda$ is applied to the points of $X_{n}$ and the convex hull of their images is constructed. If points $x_{1}$ through $x_{d+1}$ form a nearest-site dual simplex, the $d$-ball they define is empty, and so, too, is the corresponding halfspace in $\mathbf{R}^{d+1}$; thus its bounding hyperplane is a facet of the convex hull. If $x_{1}$ through $x_{d+1}$ form a furthest-site dual simplex, their $d$-ball contains all of $X_{n}$; then the complementary halfspace is empty and again the bounding hyperplane is a convex-hull facet. Conversely, a convex-hull facet always corresponds to either a nearest-site or a furthest-site dual simplex. Constructing a $(d+1)$-dimensional convex hull is a viable approach to the problem of constructing a $d$-dimensional Voronoi diagram. The gift-wrapping algorithm $[7,3,23]$ may be used in $\Theta\left(n\left(S_{n}+S_{n}^{*}\right)\right)$ time. Or Seidel's shelling algorithm [21] may be used in $O\left(n^{2}+\left(S_{n}+S_{n}^{*}\right) \log n\right)$, where $S_{n}$ is the number of nearest-site simplices and $S_{n}^{*}$ the number of 
furthest-site simplices in the result. In fact, it is not difficult to modify either algorithm to eliminate the $S_{n}^{*}\left(S_{n}\right)$ term if only the nearest-site (furthest-site) diagram is required.

Like the number of facets in the case of convex hulls, $S_{n}$ and $S_{n}^{*}$ can vary wildly. Seidel $[20,22]$ has shown that both $S_{n}$ and $S_{n}^{*}$ can be extremely large $-\theta(n\lfloor(d+1) / 2\rfloor)-$ in the worst case. On the other hand, it is not difficult to construct families of problem instances for which $S_{n}=\Theta(n)$. Thus probabilistic estimates of the average value of the two quantities are useful.

Meijering [17] and Gilbert [13] have considered the Voronoi diagram of sites from a Poisson process of fixed intensity in $\mathbf{R}^{d}$; Meijering showed that the expected number of nearest-site Voronoi neighbors of a site depends only on $d$; in particular, it is 6 for $d=2$ and $\approx 15.54$ for $d=3$. Such a set of sites may be thought of as an infinite set of sites drawn from a uniform distribution over all of $\mathbf{R}^{d}$. In computational practice, however, one must deal with finite sets of sites drawn from, e.g., a uniform distribution on the interior of some convex body like a hypercube or hypersphere. Two sites are neighbors if and only if they lie on the surface of some ball that contains no other site. In the Poisson case, a pair of distant neighbors is always unlikely since it implies the existence of a large empty ball. In the case of a bounded set of sites, it can still be shown that sites far from the boundary of the body probably have only nearby neighbors, but some long edges always occur near the boundary of the body, where most of the empty ball may lie outside the support of the distribution. Thus results dealing only with the Poisson case are insufficient for the average-case analysis of algorithms.

In the next section, we present a new method for determining $E S_{n}$ and $E S_{n}^{*}$. In $\S 3$ this method is applied to the analysis of the asymptotic behavior of $E S_{n}$ for sites drawn independently from the uniform distribution in the unit $d$-ball. In $\S 4$ we use a similar method to show that the Voronoi diagram of such sets of sites can be constructed in linear expected time by a variation of the gift-wrapping algorithm using standard bucketing techniques.

\section{A General Method for Bounding the Expected Complexity of Voronoi Diagrams}

In this section we describe a general method for bounding $E S_{n}$ and $E S_{n}^{*}$, the expected number of simplices in the duals of nearest- and furthest-site Voronoi diagrams of random point sets.

The first $d+1$ points $x_{1}, \ldots, x_{d+1}$ define a $d$-simplex with probability one. Let us first reckon the probability that they also define a simplex in the dual of the nearest-site Voronoi diagram. This is just the probability that the other $n-d-1$ points lie outside the hypersphere passing through the $d+1$ points. Writing $g(\cdot)$ for the density function of the $x_{i}$ and $\Gamma_{d}$ for the probability content of interior of the hypersphere, we see that this probability is

$$
P_{n}=\int_{\mathbf{R}^{d}} \cdots \int_{\mathbf{R}^{d}}\left(1-\Gamma_{d}\right)^{n-d-1} g\left(x_{1}\right) \cdots g\left(x_{d+1}\right) d x_{1} \cdots d x_{d+1},
$$

and that the expected number of simplices is therefore

$$
E S_{n}=\left(\begin{array}{c}
n \\
d+1
\end{array}\right) P_{n}=\left(\begin{array}{c}
n \\
d+1
\end{array}\right) \int_{\mathbf{R}^{d}} \cdots \int_{\mathbf{R}^{d}}\left(1-\Gamma_{d}\right)^{n-d-1} g\left(x_{1}\right) \cdots g\left(x_{d+1}\right) d x_{1} \cdots d x_{d+1} .
$$

We next carry out a transformation of coordinates. The $d+1$ points $x_{1}, x_{2}, \ldots, x_{d+1}$ can be expressed in terms of a $d$-vector $p$ representing the center of the sphere they define, a scalar 
$r$ representing the radius of that sphere, and $d-1$ angles $\psi_{i 1}, \psi_{i 2}, \ldots, \psi_{i, d-1}$ for each $x_{i}$. Let $y_{i}=x_{i}-p$. Then the two systems of coordinates are related by the following equations:

$$
\begin{aligned}
x_{i}^{(1)} & =p^{(1)}+y_{i}^{(1)}=p^{(1)}+r c_{i, d-1} c_{i, d-2} \cdots c_{i 3} c_{i 2} c_{i 1} \\
x_{i}^{(2)} & =p^{(2)}+y_{i}^{(2)}=p^{(2)}+r c_{i, d-1} c_{i, d-2} \cdots c_{i 3} c_{i 2} s_{i 1} \\
x_{i}^{(3)} & =p^{(3)}+y_{i}^{(3)}=p^{(3)}+r c_{i, d-1} c_{i, d-2} \cdots c_{i 3} s_{i 2} \\
& \vdots \\
x_{i}^{(d)} & =p^{(d)}+y_{i}^{(d)}=p^{(d)}+r s_{i, d-1},
\end{aligned}
$$

\begin{tabular}{|c|c|c|c|c|c|c|c|c|c|c|c|c|}
\hline & $p^{(1)}$ & $p^{(2)}$ & $p^{(3)}$ & $r$ & $\psi_{11}$ & $\psi_{12}$ & $\psi_{21}$ & $\psi_{32}$ & $\psi_{31}$ & $\psi_{32}$ & $\psi_{\Delta 1}$ & $\psi_{42}$ \\
\hline$x_{1}^{11}$ & 1 & 0 & 0 & $y_{1}^{(2)} / r$ & $t_{11}^{\prime} y_{1}^{(1)}$ & $t_{12}^{\prime} y_{1}^{(1)}$ & 0 & 0 & 0 & 0 & 0 & 0 \\
\hline & 1 & 0 & 0 & $y_{2}^{(1)} / r$ & 0 & 0 & $t_{21}^{\prime} y_{2}^{(1)}$ & $t_{22}^{\prime} y_{2}^{(1)}$ & 0 & 0 & 0 & 0 \\
\hline & 1 & 0 & 0 & $y_{s}^{(1)} / r$ & 0 & 0 & 0 & 0 & $t_{31}^{\prime} y_{3}^{(1)}$ & $t_{\mathrm{s} 2}^{\prime} y_{3}^{(1)}$ & 0 & 0 \\
\hline$x_{4}^{(1)}$ & 1 & 0 & 0 & $y_{s}^{(1)} / r$ & 0 & 0 & 0 & 0 & 0 & 0 & $t_{41}^{\prime} y_{4}^{(1)}$ & $t_{42}^{\prime} y_{6}^{(1)}$ \\
\hline$x_{1}^{(2)}$ & 0 & 1 & 0 & $y_{1}^{(2)} / r$ & $k_{11} y_{1}^{(2)}$ & $t_{12}^{\prime} y_{1}^{(2)}$ & 0 & 0 & 0 & 0 & 0 & 0 \\
\hline$x_{1}^{(3)}$ & 0 & 0 & 1 & $y_{1}^{(3)} / r$ & 0 & $k_{12} y_{1}^{(3)}$ & 0 & 0 & 0 & 0 & 0 & 0 \\
\hline$x_{2}^{(2)}$ & 0 & 1 & 0 & $y_{2}^{(2)} / r$ & 0 & 0 & $k_{21} y_{2}^{(2)}$ & $t_{22}^{\prime} y_{2}^{(2)}$ & 0 & 0 & 0 & 0 \\
\hline$x_{2}^{78}$ & 0 & 0 & 1 & $y_{2}^{(s)} / r$ & 0 & 0 & 0 & $k_{22} y_{2}^{(3)}$ & 0 & 0 & 0 & 0 \\
\hline$x_{3}^{i}$ & 0 & 1 & 0 & $y_{s}^{(2)} / r$ & 0 & 0 & 0 & 0 & $k_{31} y_{3}^{(2)}$ & $t_{82}^{\prime} y_{3}^{(2)}$ & 0 & 0 \\
\hline$x_{0}$ & 0 & 0 & 1 & $y_{8}^{(3)} / r$ & 0 & 0 & 0 & 0 & 0 & $k_{32} y_{3}^{(3)}$ & 0 & 0 \\
\hline$x_{4}^{(2)}$ & 0 & 1 & 0 & $y_{4}^{(2)} / r$ & 0 & 0 & 0 & 0 & 0 & 0 & $k_{41} y_{4}^{(2)}$ & $t_{12}^{\prime} y_{t}^{(2)}$ \\
\hline$x_{4}^{(3)}$ & 0 & 0 & 1 & $y_{4}^{(3)} / r$ & 0 & 0 & 0 . & 0 & 0 & 0 & 0 & $k_{42} y_{4}^{(s)}$ \\
\hline
\end{tabular}

where $c_{i j}$ and $s_{i j}$ represent $\cos \psi_{i j}$ and $\sin \psi_{i j}$ respectively.

In three dimensions, the Jacobian of this transformation, expressed in tabular form, is

where $t_{i j}^{\prime}=-\tan \theta_{i j}$ and $k_{i j}=\cot \theta_{i j}$. The generalization to higher dimensions is straightforward.

If the row for $x_{i}^{(1)}$, denoted by $\rho_{i}^{(1)}$, is replaced by $\sigma_{i}=\sum_{1 \leq j \leq d}\left(y_{i}^{(j)} / y_{i}^{(1)}\right) \rho_{i}^{(j)}$, it becomes

$$
y_{i}^{(1)} / y_{i}^{(1)} y_{i}^{(2)} / y_{i}^{(1)} y_{i}^{(3)} / y_{i}^{(1)} \quad r / y_{i}^{(1)} \quad 0 \quad 000000000
$$

and the matrix is in quasi-triangular form. The determinant of the entire matrix is the product of the determinant of one $(d+1) \times(d+1)$ matrix and $d+1$ similar $(d-1) \times(d-1)$ matrices.

The $(d-1) \times(d-1)$ matrices are themselves upper triangular; the determinant of each is easily seen to be

$$
r^{d-1} c_{i, d-1}^{d-1} c_{i, d-2}^{d-2} \cdots c_{i 1}^{1}=y_{i}^{(1)} r^{d-2} c_{i, d-1}^{d-2} c_{i, d-2}^{d-3} \cdots c_{i 2}^{1} .
$$

If the factors of $\left(1 / y_{i}^{(1)}\right)$ are removed from the rows of the $(d+1) \times(d+1)$ matrix, and then the factor of $r$ is removed from the last column, the remaining determinant is

$$
\left|\begin{array}{llll}
y_{1}^{(1)} & y_{1}^{(2)} & y_{1}^{(3)} & 1 \\
y_{2}^{(1)} & y_{2}^{(2)} & y_{2}^{(3)} & 1 \\
y_{3}^{(1)} & y_{3}^{(2)} & y_{3}^{(3)} & 1 \\
y_{4}^{(1)} & y_{4}^{(2)} & y_{4}^{(3)} & 1
\end{array}\right| \text {, }
$$

or just $d !$ times the volume of the simplex formed by $x_{1}, x_{2}, \ldots, x_{d+1}$. Thus the determinant of the original Jacobian matrix is

$$
d ! \operatorname{simp}\left(x_{1}, x_{2}, \ldots, x_{d+1}\right) r^{(d+1)(d-2)+1} \prod_{1 \leq i \leq d+1} c_{i, d-1}^{d-2} c_{i, d-2}^{d-3} \cdots c_{i 2}^{1}
$$


and

$$
\begin{aligned}
& P_{n}= \\
& d ! \int_{\mathbf{R}^{d}} \int_{0}^{\infty} r^{-d}\left(1-\Gamma_{d}\right)^{n-d-1}[\underbrace{\left.\int_{0}^{\pi} \cdots \int_{0}^{\pi} h\left(x_{1}\right) \cdots h\left(x_{d+1}\right) \operatorname{sim} p\left(x_{1}, \cdots, x_{d+1}\right) d \psi_{1,1} \cdots d \psi_{d+1, d-1}\right] d r d p}_{(d+1)(d-1)}
\end{aligned}
$$

with

$$
h\left(x_{i}\right)=r^{d-1} c_{i, d-1}^{d-2} c_{i, d-2}^{d-3} \cdots c_{i 2}^{1} g\left(x_{i}\right)
$$

If we define

$$
\hat{g}(r, p)=\underbrace{\int_{0}^{\pi} \cdots \int_{0}^{\pi}}_{(d-1)} h\left(x_{1}\right) d \psi_{1,1} \cdots d \psi_{1, d-1},
$$

then, since $x_{1}$ through $x_{d+1}$ are i.i.d.,

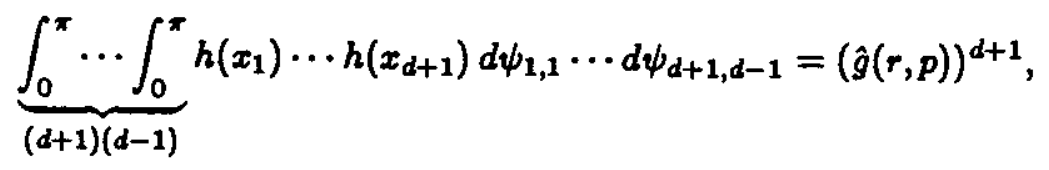

and the bracketed quantity of (2.1) is easily seen to be

$$
(\hat{g}(r, p))^{d+1} E\left(\operatorname{sim} p\left(x_{1}, x_{2}, \ldots, x_{d+1}\right) \mid\left\|x_{i}-p\right\|=r \text { for } 1 \leq i \leq d+1\right)=(\hat{g}(r, p))^{d+1} \text { esimp }(r, p) .
$$

and

$$
P_{n}=d ! \int_{\mathbf{R}^{d}} \int_{0}^{\infty} r^{-d}\left(1-\Gamma_{d}\right)^{n-d-1}(\hat{g}(r, p))^{d+1} \operatorname{esim} p(r, p) d r d p .
$$

It is clear that $\hat{g}, \Gamma_{d}$, and esimp depend only on $r$ and $\|p\|$ if $g$ is spherically symmetric. To exploit this symmetry, we express $p$ in generalized spherical coordinates $\left(q, \theta_{1}, \theta_{2}, \ldots, \theta_{d-1}\right)$ defined by

$$
\begin{aligned}
\|p\| & =q \\
p^{(1)} & =q c_{d-1} c_{d-2} \cdots c_{3} c_{2} c_{1} \\
p^{(2)} & =q c_{d-1} c_{d-2} \cdots c_{3} c_{2} s_{1} \\
p^{(3)} & =q c_{d-1} c_{d-2} \cdots c_{3} s_{2} \\
& \vdots \\
p^{(d)} & =q s_{d-1},
\end{aligned}
$$

where $c_{i}$ and $s_{i}$ represent $\cos \theta_{i}$ and $\sin \theta_{i}$. The Jacobian of this transformation is well known to be $q^{d-1} c_{d-1}^{d-2} c_{d-2}^{d-3} \cdots c_{2}^{1}[15$, p. 17], thus by application of a well-known definite-integral identity [2, $\left.\int 620\right]$ with $V_{d}=\left(2 \pi^{d / 2}\right) /(d \Gamma(d / 2))$ being the volume of the unit $d$-ball, $P_{n}$ is

$$
\begin{aligned}
& d !(\underbrace{\left.\int_{0}^{\pi} \cdots \int_{0}^{\pi} \int_{0}^{2 \pi} c_{d-1}^{d-2} c_{d-2}^{d-3} \cdots c_{2}^{1} d \theta_{1} \cdots d \theta_{d-1}\right)\left(\int_{0}^{\infty} \int_{0}^{\infty} q^{d-1} r^{-d}\left(1-\Gamma_{d}\right)^{n-d-1} \hat{g}^{d+1} e s i m p d r d q\right)}_{(d-2)} \\
& \sim d ! d V_{d} \cdot \int_{0}^{\infty} \int_{0}^{\infty} q^{d-1} r^{-d} \hat{g}^{d+1} \operatorname{esimp} \exp \left(-n \Gamma_{d}\right) d r d q .
\end{aligned}
$$


and, since $\left(\begin{array}{c}n \\ d+1\end{array}\right) \sim \frac{n^{d+1}}{(d+1)^{!}}$,

$$
E S_{n} \sim \frac{d V_{d} n^{d+1}}{d+1} \cdot \int_{0}^{\infty} \int_{0}^{\infty} q^{d-1} r^{-d} \hat{g}^{d+1} \operatorname{esimp} \exp \left(-n \Gamma_{d}\right) d r d q=\frac{d V_{d} n^{d+1}}{d+1} \cdot \int_{0}^{\infty} \int_{0}^{\infty} I(q, r) d r d q
$$

Similarly,

$$
E S_{n}^{*} \sim \frac{d V_{d} n^{d+1}}{d+1} \cdot \int_{0}^{\infty} \int_{0}^{\infty} q^{d-1} r^{-d} \hat{g}^{d+1} \operatorname{esimp} \exp \left(-n\left(1-\Gamma_{d}\right)\right) d r d q
$$

\section{Bounds for The Uniform Distribution in a $d$-Ball}

In this section we turn to the uniform distribution in the unit $d$-ball in particular and prove the following theorem.

Theorem 3 Let $X_{n}=\left\{X_{1}, X_{2}, \ldots, X_{n}\right\}$ be a set of $n$ sites drawn independently from the uniform distribution on the interior of the unit d-ball. Then $E S_{n}$, the expected number of simplices of the dual of the Voronoi diagram of $X_{n}$, is $\Theta(n)$.

Proof. We have $g(x)=1 / V_{d}$ when $\|x\| \leq 1$ and $g(x)=0$ otherwise. Let $u$ denote the unit $d$-ball, $B$ the ball defined by the points $x_{1}$ through $x_{d+1}$, and $\partial B$ the surface of $B$. Then

$$
\begin{aligned}
\Gamma_{d} & =\frac{\operatorname{vol}(B \cap U)}{V_{d}}, \\
\hat{g} & =\frac{\operatorname{vol}_{d-1}(\partial B \cap U)}{V_{d}}, \\
\text { esimp } & \leq \operatorname{vol}(\operatorname{conv}(\partial B \cap U)) .
\end{aligned}
$$

We now divide the domain of integration of the integral of (2.2) into eight regions corresponding to the possible patterns of intersection of the two balls $B$ and $U$.

Case 1: $q \leq 1$ and $0 \leq r \leq 1-q$. In this case, $B \subset U$,

$$
\hat{g}=\frac{S_{d} r^{d-1}}{V_{d}}=d r^{d-1} ; \quad \text { and } \quad \Gamma_{d}=\frac{V_{d} r^{d}}{V_{d}}=r^{d}
$$

Also

$$
\operatorname{esimp}=M_{d} r^{d}
$$

we return to the exact value of $M_{d}$ later. Thus

$$
\begin{aligned}
\int_{0}^{1} \int_{0}^{1-q} I(q, r) d r d q & \sim d^{d+1} M_{d} \int_{0}^{1} \int_{0}^{1-q} q^{d-1} r^{d^{2}-1} \exp \left(-n r^{d}\right) d r d q \\
& \sim d^{d+1} M_{d}\left(\int_{0}^{1} q^{d-1} d q\right)\left(\frac{1}{d} \int_{0}^{n(1-q)^{d}}\left(\frac{t}{n}\right)^{d} \frac{e^{-t}}{t} d t\right) \\
& \sim d^{d-2} d ! M_{d} n^{-d} .
\end{aligned}
$$




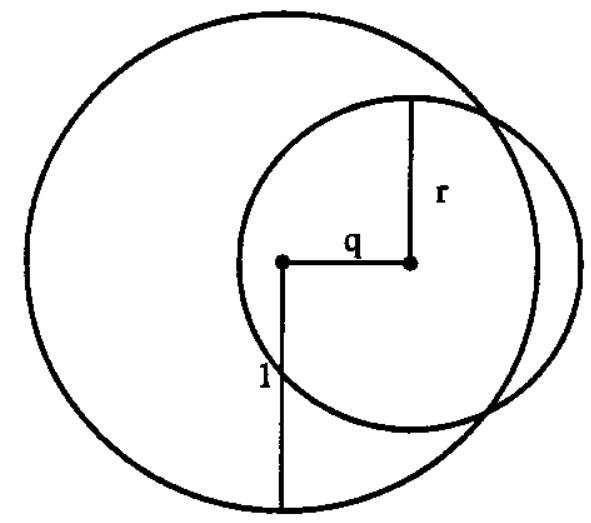

Figure 1: Case 2: $q \leq 1$ and $1-q \leq r \leq \sqrt{1-q^{2}}$.

Case 2: $q \leq 1$ and $1-q \leq r \leq \sqrt{1-q^{2}}$. In this case at least half of $B$ and $\partial B$ lie inside $U$, and $\hat{g} \leq \frac{S_{d} r^{d-1}}{V_{d}}=d r^{d-1} ; \quad \Gamma_{d} \geq \frac{V_{d} r^{d}}{2 V_{d}}=\frac{r^{d}}{2} ; \quad$ esimp $\leq V_{d} r^{d}$.

By Tricomi's formula $\int_{x}^{\infty} t^{-a} e^{-t} d t \sim x^{-a} e^{-x}$ for the incomplete gamma function [27, $\left.\S 4.3\right]$,

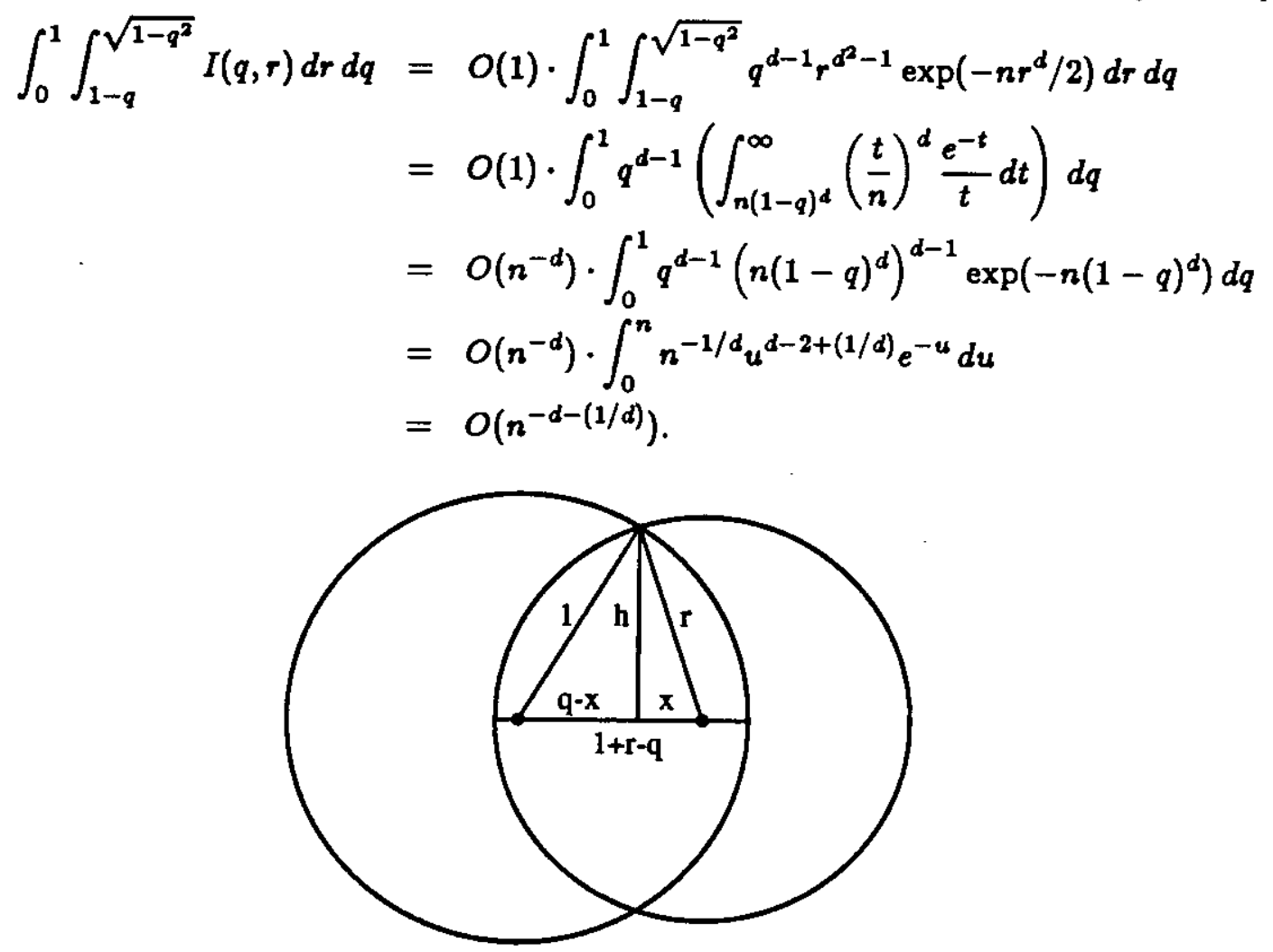

Figure 2: Case 3: $q \leq 1$ and $\sqrt{1-q^{2}} \leq r \leq 1$.

Case $8: q \leq 1$ and $\sqrt{1-q^{2}} \leq r \leq 1$. Referring to Figure 2, we have immediately from geometric considerations that $(r-x) \leq h \leq r$. By first solving the equation $1-(q-x)^{2}=r^{2}-x^{2}$ for $x$, it is easy to verify that

$$
(r-x)=\frac{(q+1-r)(r+1-q)}{2 q}
$$


Since $r \geq \sqrt{1-q^{2}} \geq 1-q$, it follows that

$$
\begin{aligned}
& r \leq(r+1-q) \leq 2 r \\
& q \leq(q+1-r) \leq 2 q
\end{aligned}
$$

thus $(r-x)=\theta(r)$ and also $h=\theta(r)$. Now

$$
\begin{aligned}
\hat{g} & =\frac{S_{d} r^{d-1}}{V_{d}} S\left(\frac{x}{r}\right)=\Theta(1) r^{d-1}\left(1-\frac{x}{r}\right)^{(d-1) / 2}=\Theta\left(r^{d-1}\right) ; \\
\Gamma_{d} & \geq \frac{(r+1-q) V_{d-1} h^{d-1}}{d V_{d}}=\Theta\left(r^{d}\right) \\
\text { esimp } & \leq(r-x) V_{d-1} h^{d-1}=\Theta\left(r^{d}\right),
\end{aligned}
$$

and

$$
\begin{aligned}
\int_{0}^{1} \int_{\sqrt{1-q^{2}}}^{1} I(q, r) d r d q & =\theta(1) \int_{0}^{1} \int_{\sqrt{1-q^{2}}}^{1} q^{d-1} r^{-d} r(d+1)(d-1) / 2 r^{d} \exp \left(-n r^{d}\right) d r d q \\
& =\theta(1) \int_{0}^{1} q^{d-1}\left(\int_{n\left(1-q^{2}\right)^{d / 2}}^{n}\left(\frac{t}{n}\right)^{d} e^{-t} \frac{d t}{t}\right) d q
\end{aligned}
$$

this is clearly dominated by (3.1) of Case 2 .

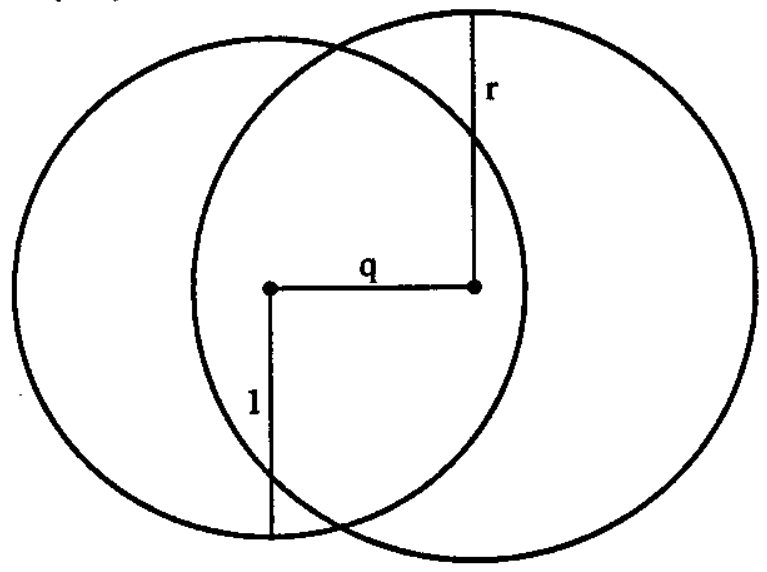

Figure 3: Case 4: $q \leq 1$ and $r \geq 1$.

Case 4: $q \leq 1$ and $r \geq 1$. In this case

$$
\begin{aligned}
\hat{g} & \leq S_{d} / V_{d}=d \\
\Gamma_{d} & \geq 2 \operatorname{vol}\left(u \cap\left\{x \mid x^{(1)} \geq 1 / 2\right\}\right)=\Omega(1) \\
\text { esimp } & \leq V_{d}
\end{aligned}
$$

and

$$
\int_{0}^{1} \int_{1}^{\infty} I(q, r) d r d q \leq \int_{0}^{1} \int_{1}^{\infty} q^{d-1} r^{-d} d^{d+1} V_{d} \exp (-\Omega(n)) d r d q=O\left(e^{-\Omega(n)}\right)
$$

Case 5: $q>1$ and $0 \leq r \leq q-1$. In this case $B \cap U=0, \hat{g}=\Gamma=e s i m p=0$, and the integral vanishes. 


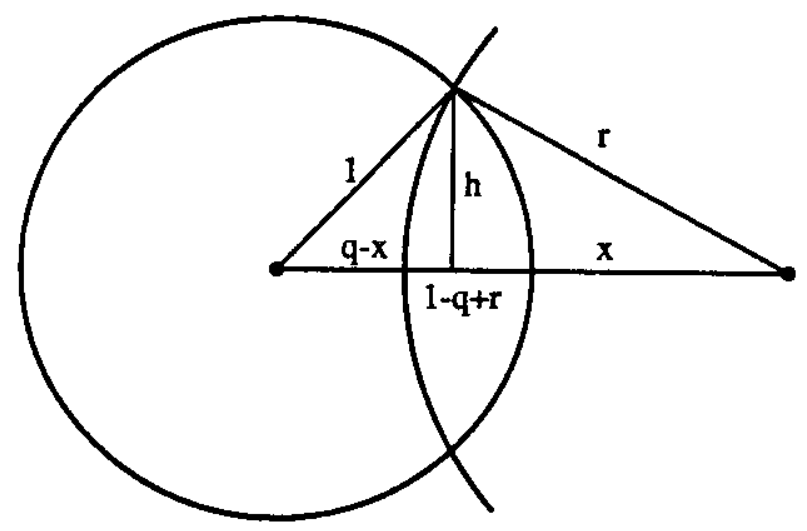

Figure 4: Case 6: $q>1$ and $q-1 \leq r \leq q$.

Case 6: $q>1$ and $q-1 \leq r \leq q$. Setting $w=1-q+r$, we have

$$
\begin{aligned}
x & =\frac{r^{2}+q^{2}-1}{2 q}=r-\frac{(2-w) w}{2 q}=r-\theta(w / q) \\
h & =\frac{1}{2 q} \sqrt{-(1+q+r)(1+q-r)(1-q+r)(1-q-r)} \\
& =\frac{1}{2 q} \sqrt{(2 q+w)(2-w) w(r+q-1)} \\
& =\theta\left(\sqrt{\frac{w r}{q}}\right)
\end{aligned}
$$

since

$$
\begin{gathered}
0 \leq w \leq 1 \\
1 \leq(2-w) \leq 2 \\
2 q \leq(2 q+w) \leq 3 q \\
r \leq(r+q-1) \leq 2 r
\end{gathered}
$$

Thus

$$
\begin{aligned}
\Gamma & =\Theta\left(w h^{d-1}\right) \\
\operatorname{esimp} & \leq V_{d-1} h^{d-1}(r-x)=\Theta(\Gamma / q) \\
\hat{g} & =\frac{S_{d} r^{d-1}}{V_{d}} S(x / r)=\Theta\left(r^{d-1}(1-(x / r))^{(d-1) / 2}\right)=\theta\left((w r / q)^{(d-1) / 2}\right)=\theta(\Gamma / w) .
\end{aligned}
$$

Now

We have

$$
\begin{aligned}
\int_{1}^{\infty} \int_{q-1}^{q} I(q, r) d r d q & =\Theta(1) \int_{1}^{\infty} \int_{q-1}^{q}\left(\frac{q^{d-1}}{r^{d}}\right)\left(\frac{\Gamma}{w}\right)^{d+1}\left(\frac{\Gamma}{q}\right) e^{-n \Gamma} d r d q \\
& =\Theta(1)\left(\int_{1}^{\infty} q^{-2} d q\right)\left(\int_{0}^{1} w^{-1}\left(\frac{q}{w r}\right)^{d} \Gamma^{d+2} e^{-n \Gamma} d w\right)
\end{aligned}
$$

$$
\begin{aligned}
& \frac{d h}{d w}=\frac{(w+r)}{2 q} \sqrt{\frac{q}{w r}}=\Theta\left(\frac{h}{w}\right) \\
& \frac{d \Gamma}{d w}=h^{d-1}+w(d-1) h^{d-2}\left(\frac{d h}{d w}\right)=\Theta\left(h^{d-1}\right)=\Theta(\Gamma / w)
\end{aligned}
$$


Substituting $t=n \Gamma ; d t=\Theta(t / w) d w$ gives

$$
\begin{aligned}
\int_{1}^{\infty} \int_{q-1}^{q} I(q, r) d r d q & =\Theta\left(n^{-(d+2)}\right) \int_{0}^{n} h^{-2 d} t^{d+1} e^{-t} d t \\
& =\Theta\left(n^{-(d+2)}\right) \int_{0}^{n}\left(\frac{w}{h \Gamma}\right)^{2} t^{d+1} e^{-t} d t \\
& =\Theta\left(n^{-d}\right) \int_{0}^{n}\left(\frac{w}{h}\right)^{2} t^{d-1} e^{-t} d t
\end{aligned}
$$

Since the $\Gamma$-region contains a ball of radius $w / 2, \Theta\left(w h^{d-1}\right)=\Gamma \geq \Omega\left(w^{d}\right)$ and $w / h=O(1)$, and

$$
\int_{1}^{\infty} \int_{q-1}^{q} I(q, r) d r d q=O\left(n^{-d}\right)
$$

A more careful analysis would show that $w / h$ is small unless $q \approx 1$. The ultimate result would be

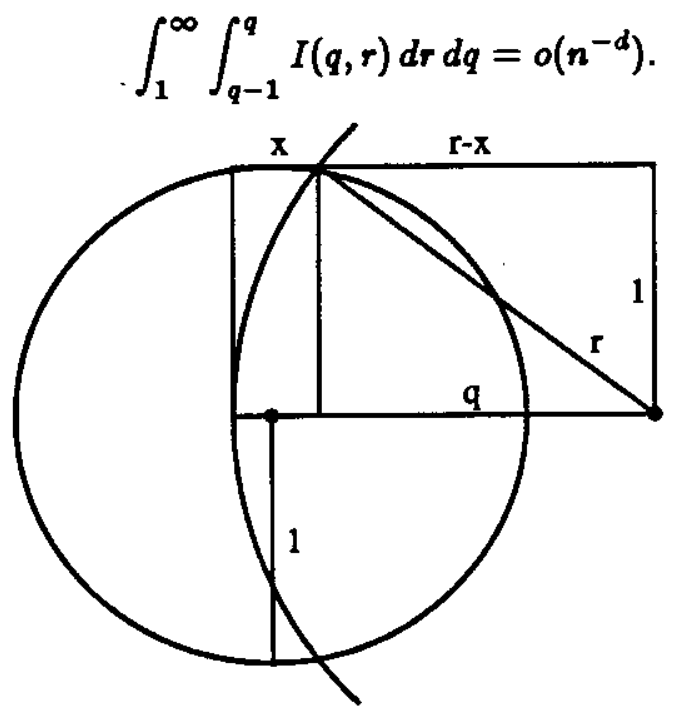

Figure 5: Case 7: $q>1$ and $q \leq r \leq q+1$.

Case 7: $q>1$ and $q \leq r \leq q+1$.

$\hat{g} \leq S_{d} / V_{d}=d ; \quad \Gamma_{d}=\Omega(1) ; \quad$ esimp $\leq V_{d-1} 2^{d-1} x=V_{d-1} 2^{d-1}\left(r-\sqrt{r^{2}-1}\right)=O\left(r^{-1}\right)$.

Thus, since $r^{-1} \leq q^{-1}$,

$$
\begin{aligned}
\int_{1}^{\infty} \int_{q}^{q+1} I(q, r) d r d q & =\int_{1}^{\infty} \int_{q}^{q+1} q^{d-1} r^{-d} O(1) O\left(r^{-1}\right) \exp (-n \Omega(1)) d r d q \\
& =\left(\int_{1}^{\infty} q^{-2} d q\right)\left(\int_{q}^{q+1} d r\right) O\left(e^{-\Omega(n)}\right) \\
& =O\left(e^{-n(n)}\right) .
\end{aligned}
$$

Case 8: $q>1$ and $q+1 \leq r$. In this case $u \subset B$ and $\partial B \cap U=\emptyset$, thus $\hat{g}=0$ and the integral vanishes.

Examining all the cases, we see that Case 1 dominates, and that

$$
E S_{n} \sim \frac{d ! d^{d-1} V_{d} M_{d} n}{(d+1)}
$$


The quantity $M_{d}$ is simply the expected volume of the simplex defined by $d+1$ points chosen at random on the surface of the unit $d$-ball. This quantity has been investigated by Miles [18], who showed that

$$
M_{d}=\frac{\Gamma\left(\left(d^{2}+1\right) / 2\right) \Gamma(d / 2)^{d+1}}{\sqrt{\pi} d ! \Gamma\left(d^{2} / 2\right) \Gamma((d+1) / 2)^{d}} .
$$

Applying (3.3) for $d=2$, we obtain $E S_{n} \sim 2 n$. This is confirmed by well-known combinatorial results. We also have

$$
\begin{array}{ll}
E S_{n} \sim \frac{24 \pi^{2}}{35} n \approx 6.77 n & \text { for } d=3 \\
E S_{n} \sim \frac{286}{9} n \approx 31.78 n & \text { for } d=4 .
\end{array}
$$

These values are not obviously inconsistent with the values 6.31 and 25.6 found empirically by Avis \& Bhattacharya for (rather small) samples of 1000 points chosen from the unit hypercube [1, Table 1]; it is reasonable to conjecture that (3.3) in fact holds for point sets chosen from a uniform distribution on any convex body.

\section{A Fast Algorithm for the Unit d-Ball}

It is immediate from Theorem 3 and the discussion of the lifting function in $§ 1$ that the Voronoi diagram of random points from a $d$-ball can be constructed in $O\left(n^{2}\right)$ time on average by either the shelling or the gift-wrapping algorithm. In this section, we describe an algorithm requiring only $O(n)$ time on average.

The algorithm we use constructs the Voronoi dual and is similar in spirit to Maus' planar algorithm [16]: it employs standard bucketing techniques, and its operation in $\mathbf{R}^{d}$ corresponds to the operation of the gift-wrapping algorithm in $\mathbf{R}^{d+1}$. It will be convenient to call the $d$-simplices of the Voronoi dual cells and the $(d-1)$-simplices facets (since they are facets of the cells); likewise, we will call an empty $d$-sphere defined by the vertices of a cell a cell sphere and a $(d-1)$-sphere defined by the vertices of a facet a facet sphere. The algorithm proceeds by repeatedly finding a new cell adjacent to a known facet. Except for facets that are also facets of the (d-dimensional) convex hull, every facet belongs to exactly two cells. We maintain a dictionary of facets for which only one cell is known. At each step a facet is removed from the dictionary and its unknown cell (if it exists) is found by searching for the unknown $(d+1)$ st vertex (the site search). The remaining facets of the new cell are searched for in the dictionary (facet searches). Each that is found is deleted, since both of its cells are already known. Each that is not found is inserted so that its unknown cell will be searched for in some later step. The algorithm is described more formally in Figure 6.

The facet dictionary is organized as a linear array of $n$ buckets; a random facet falls into a particular bucket with probability $1 / n$. Within each bucket facets may be organized in a balanced search tree to insure good (logarithmic) worst-case performance, but a simple linear list is sufficient to achieve a linear bound on expected time.

The pseudo-code of Figure 7 describes the searching function Find_site. To speed the site searches, we partition the hypercube $[-1,1]^{d}$ into $2^{d} n / V_{d}$ hypercubic boxes of volume $V_{d} / n$ and 


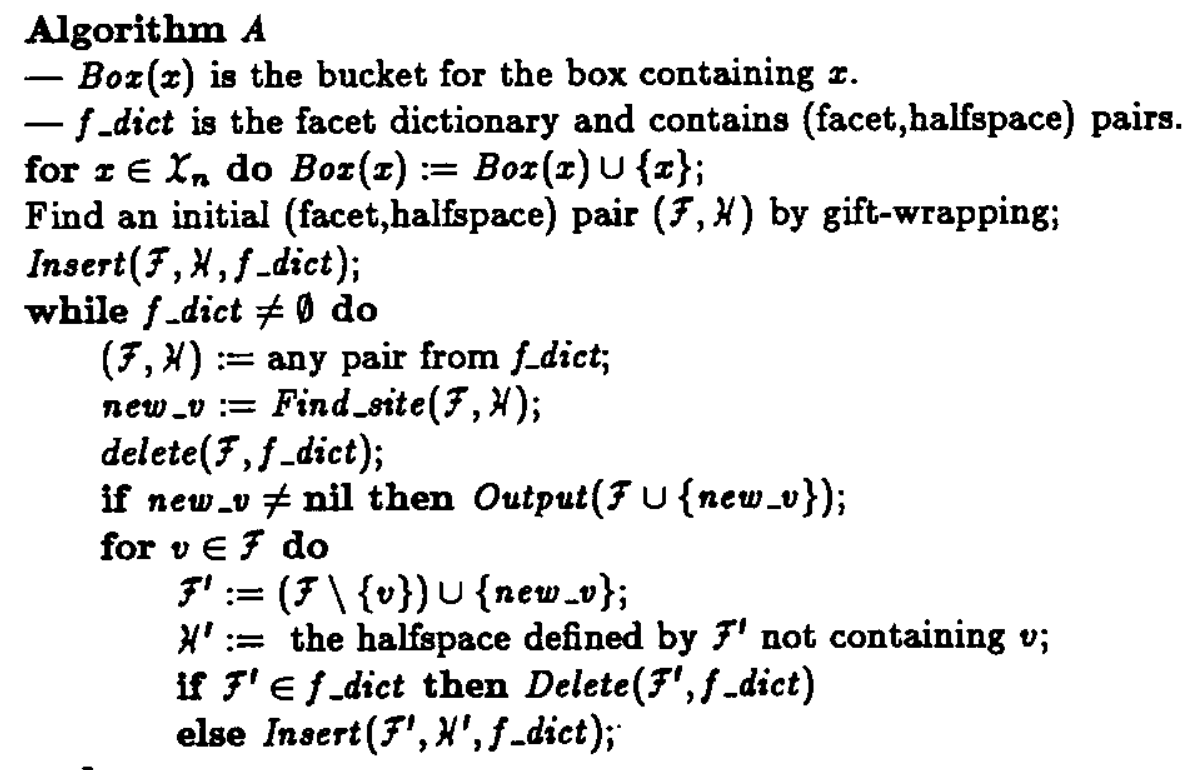

end

Figure 6: Algorithm A.

side $\left(V_{d} / n\right)^{1 / d}$ and assign each site to the bucket for the box in which it lies. Boxes lying completely outside the unit ball will always be empty. Boxes lying inside the unit ball will contain in expectation one site each. An asymptotically vanishing fraction of the boxes will intersect the boundary of the unit ball and will contain less than one site each in expectation. On a particular call to Find_site, let $\mathcal{F}, \mathcal{X}$, and $U$ be as in Figure 6 , and let $B$ be the cell ball of the unknown cell if it exists. If the cell exists, it is necessary and sufficient to examine those boxes intersecting $(B \cap \mathcal{K} \cap U)$ to determine it. If it does not exist, it is necessary and sufficient to examine the boxes intersecting $(H \cap U)$ to determine this. The function Find_site examines exactly those boxes. The priority queue operations Insert, Find_min, and Delete_min can be implemented so that only $O(\log n)$ time is required for each [25], but a naive linked-list implementation in which each operation requires time proportional to the length of the list suffices for the purposes of our average-case analysis.

We must now show that all the facet and sites searches can be completed in $O(n)$ time on average.

Lemma 4 The facet searches can be completed in $O(n)$ expected time.

Proof. The orientation of the facets with respect to the origin is obviously uniformly distributed because the distribution of sites is spherically symmetric. So we partition the surface of the unit $d$-sphere into $n$ regions of equal size and assign each facet to the bucket for the region in which its unit normal vector lies. If $u$ is the facet's unit normal vector, it will suffice, for example, to assign the facet to the bucket numbered

$$
\left\lceil\frac{2 n}{\pi} \arccos \left|\frac{u^{(1)}}{\sqrt{\left(u^{(1)}\right)^{2}+\left(u^{(2)}\right)^{2}}}\right|\right\rceil ;
$$

this assignment can be made in constant time if the ceiling function carries unit cost. Each facet will be searched for twice. Let $M_{i}$ be the number of facets ever placed into the ith bucket over the 


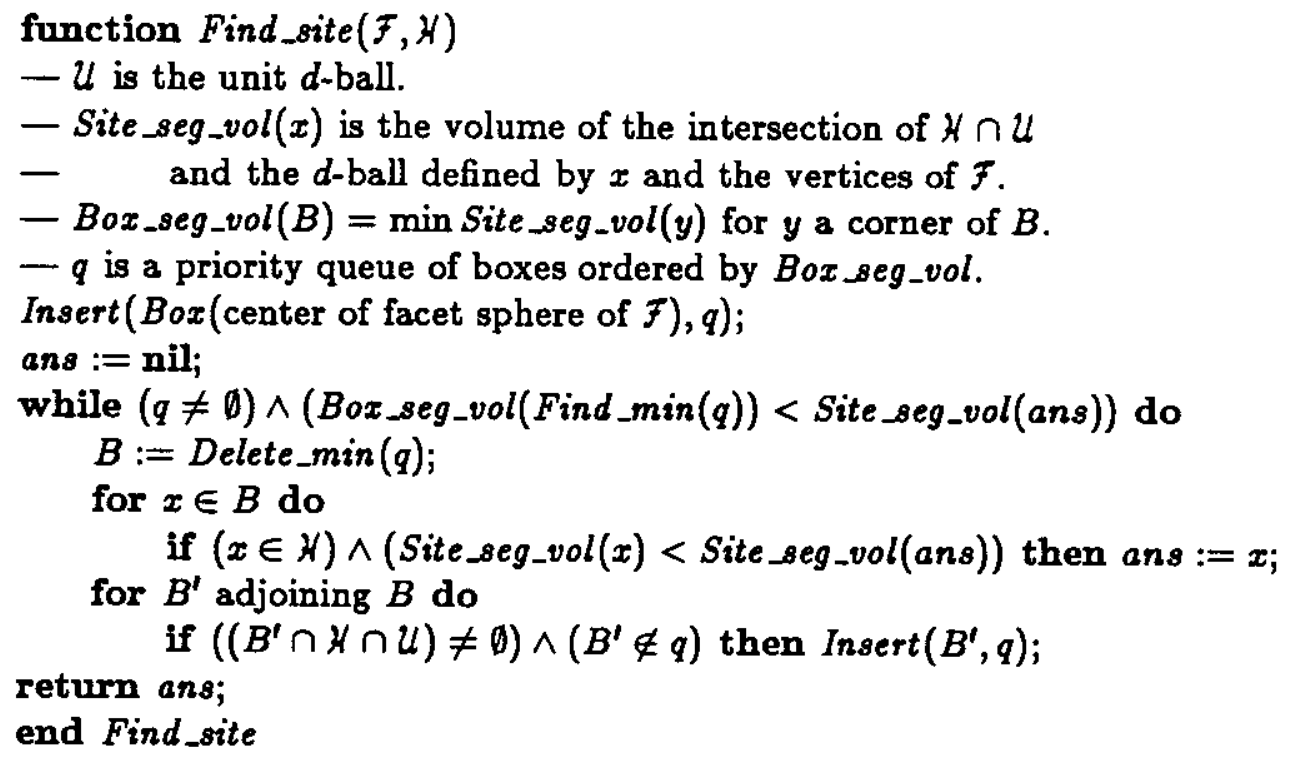

Figure 7: The site-search procedure Find_site.

course of the running of the algorithm. The time required for all the dictionary operations together (assuming linear lists within buckets) is at most

$$
\sum_{i=1}^{n} 2 M_{i}^{2} .
$$

A theorem of Devroye's [8, p. 59] asserts that the expectation of this sum is $O(n)$ if $E M_{i}=O(1)$; this is clearly the case.

We now turn to the search for the $(d+1)$ st site completing a cell with a known facet. We call a site search "successful" if a site is found, and "unsuccessful" if no site is found because the facet lies on the boundary of the convex hull.

Lemma 5 The successful site searches can be completed in $O(n)$ expected time.

Proof. If we define the distance between a point $x$ and a set $y$ by

$$
\operatorname{dist}(x, y)=\min _{y \in y} \operatorname{dist}(x, y)
$$

all the boxes intersecting $B \cap \mathcal{H} \cap U$ are completely contained by the set

$$
A=\left\{x \mid \operatorname{dist}(x, B \cap U) \leq \sqrt{d}\left(V_{d} / n\right)^{1 / d}\right\} .
$$

Assuming the naive linked-list implementation, the cost of the each priority queue operation is at most proportional to the total number of boxes examined. The cost of examining the sites in a box is $O(1)$ in expectation. The expected total cost of the site search is therefore proportional to the square of the number of boxes examined, or

$$
\text { ecost }=O\left((n \cdot \operatorname{vol} A)^{2}\right)
$$


If we write $C_{n}$ for the total cost of all successful site searches needed to compute the Voronoi diagram of $x_{n}$, we have

$$
E C_{n} \leq\left(\begin{array}{c}
n \\
d+1
\end{array}\right) \int_{\mathbf{R}^{d}} \cdots \int_{\mathbf{R}^{d}} \operatorname{ecost}\left(x_{1}, \ldots, x_{d+1}\right)\left(1-\Gamma_{d}\right)^{n-d-1} g\left(x_{1}\right) \cdots g\left(x_{d+1}\right) d x_{1} \cdots d x_{d+1} ;
$$

The $(d+1)$-fold integral represents an upper bound on the expected cost of a successful site search to complete the cell $x_{1} x_{2} \ldots x_{d+1}$. Proceeding as in $\S 2$, we eventually obtain

$$
E C_{n}=O(1) \cdot \int_{0}^{\infty} \int_{0}^{\infty} \operatorname{ecost}(q, r) I(q, r) d r d q
$$

We continue as in $\S 2$, dividing the domain of integration into eight regions. However, we ignore constant factors this time.

Cases 1, 2, 8: In all these cases,

$$
\hat{g}=\theta\left(r^{d-1}\right) ; \quad \Gamma_{d}=\theta\left(r^{d}\right) ; \quad \text { esimp }=\theta\left(r^{d}\right) .
$$

The set $A$ of (4.1) is contained in a $d$-ball of radius $\left(r+\sqrt{d}\left(V_{d} / n\right)^{1 / d}\right)^{d}$ and, by (4.2), with $t=$ $n \Gamma_{d}=\theta\left(n r^{d}\right)$ as in $\S 3$,

$$
\sqrt{\operatorname{ecost}(q, r)}=O\left(n\left(r+n^{-1 / d}\right)^{d}\right)=O\left(\sum_{i=0}^{d} r^{i} n^{i / d}\right)=O\left(\sum_{i=0}^{d} t^{i / d}\right)=O(1+t)
$$

It follows that

$$
\begin{aligned}
\int_{0}^{1} \int_{0}^{1} e \operatorname{cost}(q, r) I(q, r) d r d q & =\Theta(1)\left(\int_{0}^{1} q^{d-1} d q\right)\left(\int_{0}^{n}(1+t)^{2}\left(\frac{t}{n}\right)^{d} \frac{e^{-t}}{t} d t\right) \\
& =\Theta\left(n^{-d}\right) .
\end{aligned}
$$

Cases 4, 7: For these cases we apply the trivial bound ecost $(r, q)=O\left(n^{2}\right)$, and

$$
\begin{aligned}
\iint e \operatorname{cost}(q, r) I(q, r) d r d q & =O\left(n^{2}\right) \iint I(q, r) d r d q \\
& =O\left(e^{-n(n)}\right)
\end{aligned}
$$

Cases 5, 8: In these cases $\hat{g}=0$ and the integral vanishes as before.

Case 6: In this case the set $A$ of $(4.1)$ is contained in a $(d-1)$-spherical cylinder with height $\left(w+2 \sqrt{d}\left(V_{d} / n\right)^{1 / d}\right)$ and base radius $\left(h+\sqrt{d}\left(V_{d} / n\right)^{1 / d}\right)$, so

$$
\begin{aligned}
\sqrt{\operatorname{ecost}(q, r)} & =O\left(n\left(w+n^{-1 / d}\right)\left(h+n^{-1 / d}\right)^{d-1}\right) \\
& =O(n) \cdot\left(\sum_{i=0}^{d-1} w n^{-i / d} h^{d-1-i}+\sum_{i=1}^{d} n^{-i / d} h^{d-i}\right) \\
& =O(n) \cdot\left(w h^{d-1}+\sum_{i=1}^{d-1}(w+h) n^{-i / d} h^{d-1-i}+n^{-1}\right)
\end{aligned}
$$




$$
\begin{aligned}
& =O\left(n w h^{d-1}+\sum_{i=1}^{d-1} h^{i} n^{i / d}+1\right) \\
& =O\left(n \Gamma_{d}+\sum_{i=0}^{d-1}\left(\frac{h n \Gamma_{d}}{w}\right)^{i / d}\right) \\
& =O\left((h / w)\left(n \Gamma_{d}+1\right)\right),
\end{aligned}
$$

since $w=O(h)$ and $h=\left(h \Gamma_{d} / w\right)^{1 / d}$. Computation proceeds as in the proof of Theorem 3, substituting $t=n \Gamma_{d}$, to the point of (3.2), where, since $w=O(h)$, this time we have

$$
\begin{aligned}
\int_{1}^{\infty} \int_{q-1}^{q} e \operatorname{cost}(q, r) I(q, r) d r d q & =\Theta\left(n^{-d}\right) \int_{0}^{n} e \operatorname{cost}(q, r)\left(\frac{w}{h}\right)^{2} t^{d-1} e^{-t} d t \\
& =O\left(n^{-d}\right) \int_{0}^{n}(1+t)^{2} t^{d-1} e^{-t} d t \\
& =O\left(n^{-d}\right) .
\end{aligned}
$$

Summing over the eight cases, we see that

$$
\int_{0}^{\infty} \int_{0}^{\infty} \operatorname{ecost}(q, r) I(q, r) d r d q=O\left(n^{-d}\right)
$$

and

$$
E C_{n}=O\left(n^{d+1}\right) \cdot O\left(n^{-d}\right)=O(n)
$$

Finally, we consider the unsuccessful site searches for facets of the convex hull.

Lemma 6 The unsuccessful site searches can be completed in o(n) expected time.

Proof. An unsuccessful search requires examination of all the boxes intersecting $\mathcal{U} \cap \mathcal{U}$; these are completely contained in

As before,

$$
A=\left\{x \mid \operatorname{dist}(x, \not / \cap U) \leq \sqrt{d}\left(V_{d} / n\right)^{1 / d}\right\}
$$

$$
e \operatorname{cost}=O\left((n \cdot \operatorname{vol} A)^{2}\right)
$$

Let us write $U_{n}$ for the total cost of all unsuccessful site searches. These occur only for facets of the convex hull. We have

$$
E U_{n} \leq\left(\begin{array}{l}
n \\
d
\end{array}\right) \int_{\mathbf{R}^{d}} \cdots \int_{\mathbf{R}^{d}} e \operatorname{cost}\left(x_{1}, x_{2}, \ldots, x_{d}\right)\left(1-\Gamma_{d}\right)^{n-d} g\left(x_{1}\right) \cdots g\left(x_{d}\right) d x_{1} \cdots d x_{d} .
$$

Here $\Gamma_{d}$ is the probability content of the smaller halfspace defined by $x_{1}$ through $x_{d}$, and $\left(1-\Gamma_{d}\right)^{n-d}$ is the probability that all the other sites lie in the larger halfspace. We apply a standard change of variables used by Efron [11], Raynaud [19], the author [10], and others. Each of the $d$ vertices of $F$ is expressed in terms of $p$, the projection of the origin onto the hyperplane $H$, and an orthonormal basis for $\mathcal{X}-p$. After some computation similar to that of $\S 2$, we obtain

$$
E U_{n}=O\left(n^{d}\right) \cdot \int_{0}^{1} e \operatorname{cost}(r) \operatorname{esimp}(r)(\hat{g}(r))^{d} \exp \left(-n \Gamma_{d}\right) d r
$$




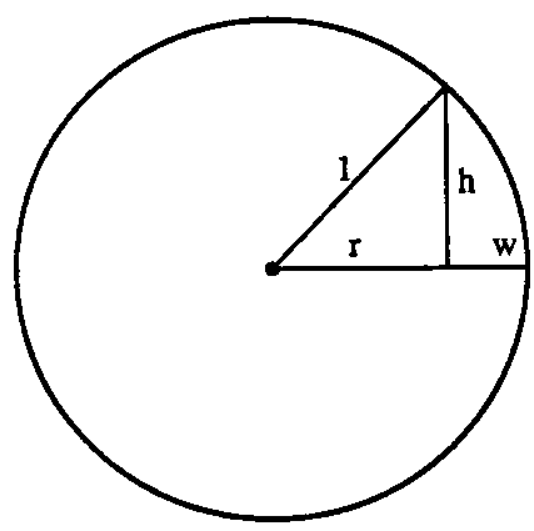

Figure 8: An unsuccessful search.

where

$r \quad$ is the distance of the hyperplane $k$ from the origin,

$\operatorname{esimp}(r)$ is the expected volume of the simplex $x_{1} x_{2} \cdots x_{d}$ given the distance $r$,

ecost $(r)$ is the expected cost of the unsuccessful search given $r$,

$\hat{g}(r)$ is the density of the probability that a random point falls on $K$, and

$\exp \left(-n \Gamma_{d}\right) \quad$ estimates the probability that $k$ defines an empty halfspace.

Let $w=1-r$ and $h=\sqrt{1-r^{2}}=\theta(\sqrt{w})$ as in Figure 8. Then

$$
\Gamma_{d}^{-}(r)=\Theta\left(w h^{d-1}\right)=\Theta\left(h^{d+1}\right)
$$

With $t=n \Gamma_{d}$ or $h=(t / n)^{1 /(d+1)}$, we have

$$
\begin{aligned}
d r & =-d w=\Theta(1)\left(\frac{t}{n}\right)^{2 /(d+1)} \frac{d t}{t} \\
e \operatorname{esimp}(r) & =\Theta\left(h^{d-1}\right)=\Theta\left((t / n)^{(d-1) /(d+1)}\right) ; \\
\hat{g}(r) & =\Theta\left(h^{d-1}\right)=\Theta\left((t / n)^{(d-1) /(d+1)}\right) ; \\
\sqrt{e \operatorname{cost}(r)} & =\Theta\left(n\left(w+n^{-1 / d}\right)\left(h+n^{-1 / d}\right)^{d-1}\right) \\
& =\Theta\left(n w h^{d-1}+\sum_{i=0}^{d-1} h^{i} n^{i / d}\right) \quad \text { as in }(4.3) \\
& =\Theta\left(t+\sum_{i=0}^{d-1}\left(t n^{1 / d}\right)^{i /(d+1)}\right) \\
& =O\left(n^{(d-1) /\left(d^{2}+d\right)}(1+t)\right)
\end{aligned}
$$

and

$$
\begin{aligned}
E U_{n} & =O\left(n^{d}\right) \int_{0}^{n}\left(n^{2(d-1) /\left(d^{2}+d\right)}(1+t)^{2}\right)\left(\frac{t}{n}\right)^{(d-1) /(d+1)}\left(\frac{t}{n}\right)^{d(d-1) /(d+1)} e^{-t}\left(\frac{t}{n}\right)^{2 /(d+1)} \frac{d t}{t} \\
& =O\left(n^{1-\left(2 /\left(d^{2}+d\right)\right)}\right) \int_{0}^{n}(1+t)^{2} t^{d-2+(2 /(d+1))} d t \\
& =o(n) . \quad \square
\end{aligned}
$$


Lemmata 4, 5, and 6 together imply the following theorem.

Theorem 7 Let $X_{n}=\left\{X_{1}, X_{2}, \ldots, X_{n}\right\}$ be a set of $n$ sites drawn independently from the uniform distribution on the interior of the unit d-ball. Then for fixed d, Algorithm A constructs the Voronoi diagram of $X_{n}$ in $O(n)$ time on average.

This algorithm is clearly optimal in the average-case sense, and is asymptotically faster than any other known. If a balanced-tree implementation of priority queues is used, the running time of this algorithm is $O\left(S_{n} n \log n\right)$, only a factor of $\Theta(\log n)$ worse that the standard gift-wrapping algorithm.

It is easy to show that linear performance is preserved if the distribution is "quasi-uniform" in the unit $d$-ball, i.e., if its density bounded above and below by a positive constant everywhere in the $d$-ball. It is an open question whether the same approach yields an $O(n)$ algorithm for other distributions. In the specific case of the uniform distribution in the unit $d$-cube, the orientation of facets is clearly not uniform, so a different approach to facet searching is required. Other distributions such as the $d$-dimensional normal likely require a different approach to site searching.

\section{Acknowledgments}

The members of my thesis committee, Danny Sleator, Ken Clarkson, Bill Eddy, Dana Scott, and Doug Tygar, all gave useful comments on drafts of this paper. Bill Eddy in particular encouraged me to push harder at this topic. Ignace Kolodner gave useful tips on determining the asymptotic behavior of the integrals. 


\section{References}

[1] D. Avis and B. K. Bhattacharya. Algorithms for computing d-dimensional Voronoi diagrams and their duals. In F. P. Preparata, editor, Advances in Computing Research: Computational Geometry, pages 159-180, JAI Press Inc., Greenwich, Conn., 1983.

[2] W. H. Beyer. CRC Standard Mathematical Tables. CRC Press, Boca Raton, Fla., 27th edition 1984.

[3] B. Bhattacharya. Worst-case analysis of a convex hull algorithm. 1982. Simon Fraser U.

[4] A. Bowyer. Computing Dirichlet tessellations. Computer J., 24(2):162-166, 1981.

[5] K. Q. Brown. Geometric Transforms for Fast Geometric Algorithms. PhD thesis, CarnegieMellon U., 1979.

[6] W. Browstow, J.-P. Dussault, and B. L. Fox. Construction of Voronoi polyhedra. J. Computational Physics, 29:81-97, 1978.

[7] D. R. Chand and S. S. Kapur. An algorithm for convex polytopes. J. Assoc. Comput. Mach., 17(1):78-86, January 1970.

[8] L. P. Devroye. Lecture Notes on Bucket Algorithms. Birkhäuser, Boston, 1986.

[9] R. A. Dwyer. A faster divide-and-conquer algorithm for constructing Delaunay triangulations. Algorithmica, 2(2):137-151, 1987.

[10] R. A. Dwyer. On the Convex Hull of Random Points in a Polytope. Technical Report CMUCS-87-118R, Carnegie-Mellon University, October 1987.

[11] B. Efron. The convex hull of a random set of points. Biometrika, 52:331-342, 1965.

[12] J. L. Finney. A procedure for the construction of Voronoi polyhedra. J. Computational Physics, 32:137-143, 1979.

[13] E. N. Gilbert. Random subdivisions of space into crystals. Ann. Math. Stat., 33:958-972, 1962.

[14] L. J. Guibas and J. Stolf. Primitives for the manipulation of general subdivisions and the computation of Voronoi diagrams. ACM Trans. Graphics, 4:74-123, 1985.

[15] M. G. Kendall. A Course in the Geometry of n Dimensions. Hafner Pub. Co., New York, 1961.

[16] A. Maus. Delaunay triangulation and the convex hull of $n$ points in expected linear time. $B I T, 24: 151-163,1984$.

[17] J. L. Meijering. Interface area, edge length, and number of vertices in crystal aggregates with random nucleation. Philips Res. Rep., 8:270-290, 1953.

[18] R. E. Miles. Isotropic random simplices. Adv. Appl. Prob., 3:353-382, 1971.

[19] H. Raynaud. Sur l'envellope convexe des nuages des points aléatoires dans $\mathbf{R}^{n}$, I. J. Appl. Prob., 7:35-48, 1970. 
[20] R. Seidel. The complexity of Voronoi diagrams in higher dimensions. In Proceedings of the 20th Annual Allerton Conference on Communication, Control, and Computing, pages 94-95, University of Illinois at Urbana-Champaign, October 1982.

[21] R. Seidel. Constructing higher-dimensional convex hulls at logarithmic cost per face. In Proc. 18th ACM Symp. on Theory of Computing, pages 404-413, ACM, May 1986.

[22] R. Seidel. On the number of faces in higher-dimensional Voronoi diagrams. In Proc. Third Ann. Symp. on Computational Geometry, pages 181-185, ACM, June 1987.

[23] G. Swart. Finding the convex hull facet by facet. J. Algorithms, 6:17-48, 1985.

[24] M. Tanemura, T. Ogawa, and N. Ogita. A new algorithm for three-dimensional Voronoi tessellation. J. Computational Physics, 51(2):191-207, August 1983.

[25] R. E. Tarjan. Data Structures and Network Algorithms. Volume 44 of CBMS-NSF Regional Conference Series in Applied Mathematics, Society of Industrial and Applied Mathematics, Philadelphia, 1983.

[26] A. H. Thiessen. Precipitation averages for large areas. Monthly Weather Review, 39:10821084, July 1911.

[27] F. G. Tricomi. Funzioni Ipergeometriche Confluente. Edizioni Cremonese, Rome, 1954.

[28] G. Voronoi. Nouvelles applications des parametres continus à la theorie des formes quadratique, deuxième mémoire: recherches sur les parallélloèdres primitifs. J. reine u. angew. Math., 134(1):198-287, May 1908.

[29] D. F. Watson. Computing the $n$-dimensional Delaunay tessellation with application to Voronoi polytopes. Computer J., 24(2):167-172, 1981.

[30] E. Wigner and F. Seitz. On the constitution of metallic sodium. Physical Reviews, 43:804-810, 1933. 